\title{
PAPER
}

\section{Wireless Distributed Computing System Using High-Gain Array Yagi-Antenna}

\author{
Kosuke Katayama $^{1}$, Takaaki Baba ${ }^{2}$ and Takashi Ohsawa ${ }^{1}$ \\ ${ }^{1}$ Graduate School of Information, Production and Systems, Waseda University \\ ${ }^{2}$ Research Center of Information, Production and Systems, Waseda University \\ 2-7 Hibikino, Wakamatsu-ku, Kitakyushu-shi, Fukuoka 808-0135, Japan \\ E-mail: kkatayama@aoni.waseda.jp,tbaba@waseda.jp, takashi.ohsawa@aoni.waseda.jp
}

\begin{abstract}
In this paper, we evaluate the system of wireless distributed computing (WDC) including a $1 \times 2$ array Yagi-antenna designed for the system. This antenna is developed on a glass-epoxy printed circuit board and its geometry is as compact as $1.6 \times 150 \times$ $200 \mathrm{~mm}^{3}$. The double rat-race balun, which also works as a power divider of the array, greatly reduces insertion losses from the RF port to driver elements of the antenna and greatly increases the operational bandwidth. As a result, the insertion loss is about $2 \mathrm{~dB}$ and the antenna operates from 2.1 to $2.5 \mathrm{GHz}$. Since the double rat-race balun also has good isolation between the ports of the arrayed driver elements, this array antenna seems to be driven with independent RF signals and achieves the high gain of $13.0 \mathrm{dBi}$. The radiation pattern of this antenna is suitable for WDC. Since the half-power beamwidth of the azimuth angle is wider than that of the elevation angle, a greater number of computational nodes on the ground surface are covered. We confirm that WDC has an enhanced computational speed when using this antenna. WDC with twelve threads achieves 550\% acceleration in comparison with the case of one thread.
\end{abstract}

Keywords: double rat-race balun, message passing interface, power divider, printed circuit board, relative permittivity

\section{Introduction}

Wireless distributed computing (WDC) is a system whose computational nodes are combined over a wireless network [1]. Although the WDC can potentially provide much computational power, its performance depends on the number of computational nodes, signal-to-noise ratio, and bandwidth of RF frontends. A straightforward way to increase the signal-to-noise ratio is to use a high-gain antenna such as a Yagi-antenna [2]. However, the high-gain antenna has a narrow beam area, as shown in Fig. 1(a). In this case, the number of computational nodes ( $\mathrm{Rx})$ is reduced along with the gain. Figure 1(b) shows a beam area that has the same gain as that in Fig. 1(a), but the beam shape is different and covers a greater number of computational nodes $(\mathrm{Rx})$ owing to the reduction in the number of director elements. Thus, the array Yagi-antenna achieves high gain with a smaller number of longitudinal antenna elements [3-6]. In [3-6], the achievements of 7.1, $11.5,12.0$, and $15.4 \mathrm{dBi}$ at around $2.4 \mathrm{GHz}$ using $1 \times 2,1$ $\times 4,1 \times 4$ and $1 \times 8$ array Yagi-antennas, respectively, were reported. In $[5,6]$, high gains were reported but their antenna bands were limited to $2.37-2.54(6.9 \%)$ and 2.36 $-2.45 \mathrm{GHz}(3.8 \%)$. The array antenna requires a power divider to feed an RF signal to the baluns. The cascaded connection of the power divider and the baluns limits the operational bandwidth because the bandwidth is an intersection of those. There are several ways to increase the bandwidth of the Yagi-antenna itself up to $45 \%$, such as using thick antenna elements [7], a bowtie-shape driver antenna $[5,8]$, and a folded dipole driver antenna [9]. Therefore, the root cause of the band limitation is a cascaded connection of the power divider and balun. In order to drive the multiple ports of the array antenna, a power divider is utilized, such as an impedance-transformed microstrip line $[6,10]$ and a Wilkinson $[4,5]$ power divider. To excite the two arms of a dipole antenna as a driver element, a balun is utilized, such as microstrip-to-doublesided parallel stripline (MS-DSPS) [3-5, 7-9], microstripto-coplanar stripline (MS-CPS) [7, 8, 10], and rat-race [11] baluns.

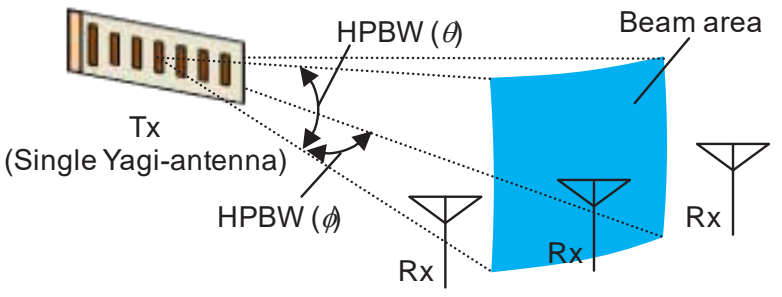

(a) Beam area of a single Yagi-antenna

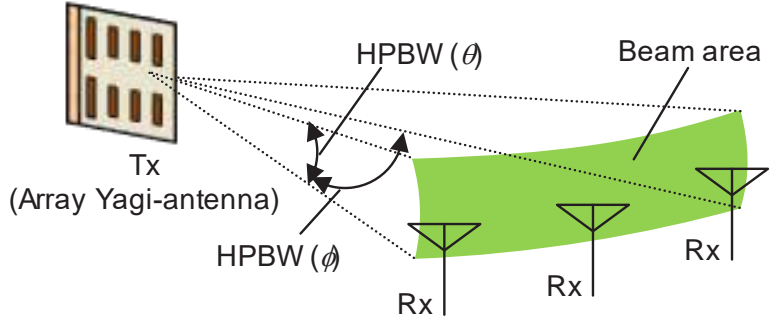

(b) Beam area of an array Yagi-antenna

Fig. 1 Coverage areas of a single Yagi-antenna and an array Yagiantenna: The array Yagi-antenna covers a greater number of computational nodes (Rx).

To avoid the combination of the power divider and balun, which limits the bandwidth, we have proposed the usage of the double rat-race balun that also works as a 
power divider $[12,13]$. In [11] a rat-race balun was also used, but it was not used as a power divider. In this paper, we propose a high-gain $1 \times 2$ array Yagi-antenna suitable for WDC on an industry science and medical (ISM) band allocated up to $2.5 \mathrm{GHz}$. In addition to [13], we estimate the profile of the printed circuit board (PCB) into which the antenna is incorporated. The estimated profile gives accurate simulation results and its estimation procedure is described in Section 2. In Section 3, we compare the single and array Yagi-antennas similarly to [13], accompanied by information of beam areas. Although the double rat-race balun has been introduced in [12, 13], we provide its measured results additionally because it is a key to this antenna. Its design details are also shown in Section 4. In Section 5, we describe the prototype of the $1 \times 2$ array Yagi-antenna on the PCB with precise simulations and measurements. The performance of WDC has been demonstrated in [13]. We provide the result of wired connected distributed computing (DC), which replaces the antenna of WDC with a wireline of gigabit Ethernet. In Section 6, we compare the WDC with this antenna prototype against DC.

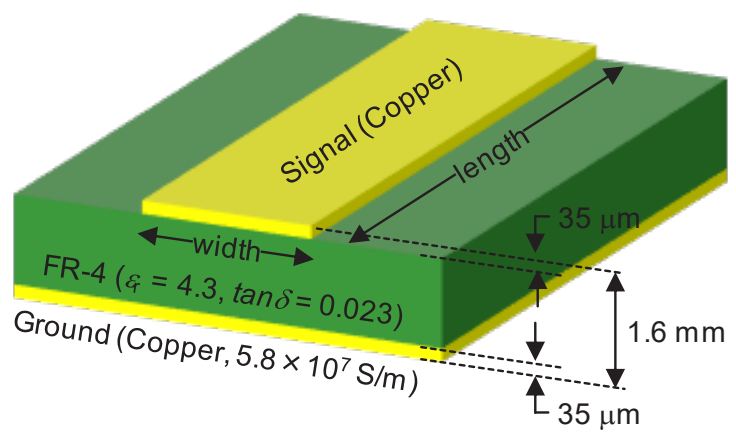

Fig. 2 Cross-sectional view of the printed circuit board (PCB): The 1.53-mm-thick glass-epoxy (FR-4) substrate is sandwiched by $35-\mu \mathrm{m}$-thick copper foils. The antenna is developed on this PCB

\section{Printed Circuit Board (PCB)}

In this study, we use a glass-epoxy (FR-4) PCB for its manufacturability and develop an antenna on it. For the precise electromagnetic (EM) simulation of an antenna, the profile of the substrate is necessary but the values at the target frequency are not given by the board manufacturer. Figure 2 shows the structure of the PCB. The 1.53mm-thick glass-epoxy (FR-4) substrate is sandwiched by $35-\mu \mathrm{m}$-thick copper foils. The relative permittivity $\left(\varepsilon_{\mathrm{r}}\right)$ and loss tangent $(\tan \delta)$ are unknown. Figure 3 shows the test element groups (TEGs) of microstrip lines. The 200mm-long microstrip lines in Figs. 3(a) and (b) are de-embedded with the 10-mm-long thru pattern in Fig. 3(c) [14]. Figure 4 shows the contour plots of root-mean-square (RMS) errors between EM-simulated $S_{21}$ and the measured $S_{21}$ results of 1.6- and 3.0-mm-wide microstrip lines while sweeping $\varepsilon_{\mathrm{r}}$ and $\tan \delta$. The minimum RMS errors are given when $\varepsilon_{\mathrm{r}}$ and $\tan \delta$ are 4.3 and 0.023 in both line widths. Figures 5(a) and (b) show measured and simulated $S_{21}$ of the microstrip lines with these estimated $\varepsilon_{\mathrm{r}}$ and $\tan \delta$ as functions of frequency. Since the measured and simulated results of $S_{21}$ are in good agreement, these estimated values are used for EM simulations in this paper hereafter.

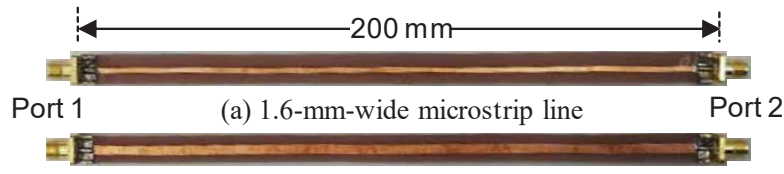

(b) 3.0-mm-wide microstrip line

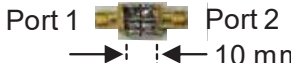

(c) Thru pattern

Fig. 3 Test element groups (TEGs) for estimation of the relative permittivity $\left(\varepsilon_{\mathrm{F}}\right)$ and loss tangent $(\tan \delta)$ : 1.6- and 3.0-mmwide transmission lines are measured and de-embedded with the thru pattern.

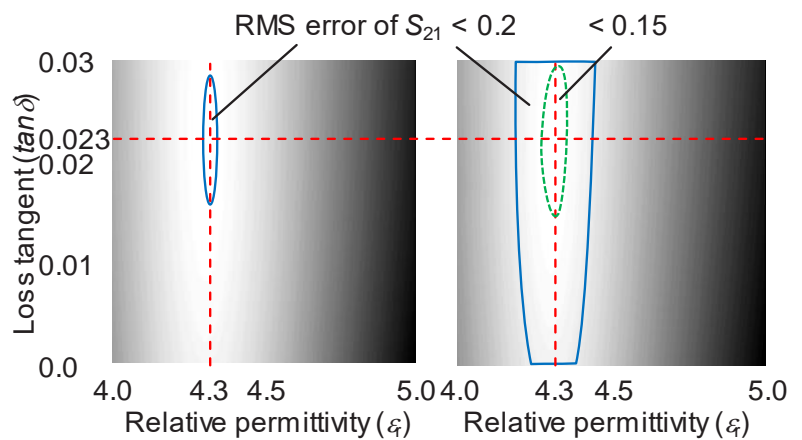

(a) Line width is $1.6 \mathrm{~mm}$

(b) Line width is $3.0 \mathrm{~mm}$

Fig. 4 Contour plots of the RMS errors between measured microstrip lines and electromagnetic (EM)-simulated ones of $S_{21}$ while changing $\varepsilon$ and $\tan \delta$. The minimum RMS errors were obtained with $\varepsilon$ and $\tan \delta$ of 4.3 and 0.023 , respectively.

\section{Single and Array Yagi-Antennas}

With the use of an array Yagi-antenna, the number of computational nodes $(\mathrm{Rx})$ is increased even though the antenna gain is equal to that of a single antenna (Fig. 1). This section provides the EM-simulated results of single and array Yagi-antennas whose geometries are shown in Fig. 6. The single Yagi-antenna is composed of reflector, driver, and director elements. The array Yagi-antenna is a pair of single Yagi-antennas, as shown in Fig. 6(b). Whether a single or array Yagi-antenna, their antenna gains are increased along with the number of director elements. Figure 7 shows the antenna gains with respect to the number of director elements. The single and array Yagi-antennas achieve $+13 \mathrm{dBi}$ with six and three director elements, respectively. However, the shapes of the beam area have different azimuth $(\phi)$ and elevation $(\theta)$ angles of the halfpower beamwidths (HPBWs), as shown in Fig. 7. Note that the azimuth angle of HPBW $(\phi)$ only depends on the number of directors regardless of the single or the array Yagi-antenna. This result supports the concept in Fig. 1. In the EM simulation, driver elements of the array Yagi-antenna are independently excited, and electrical fields $|E|$ are numerically merged on a space. 


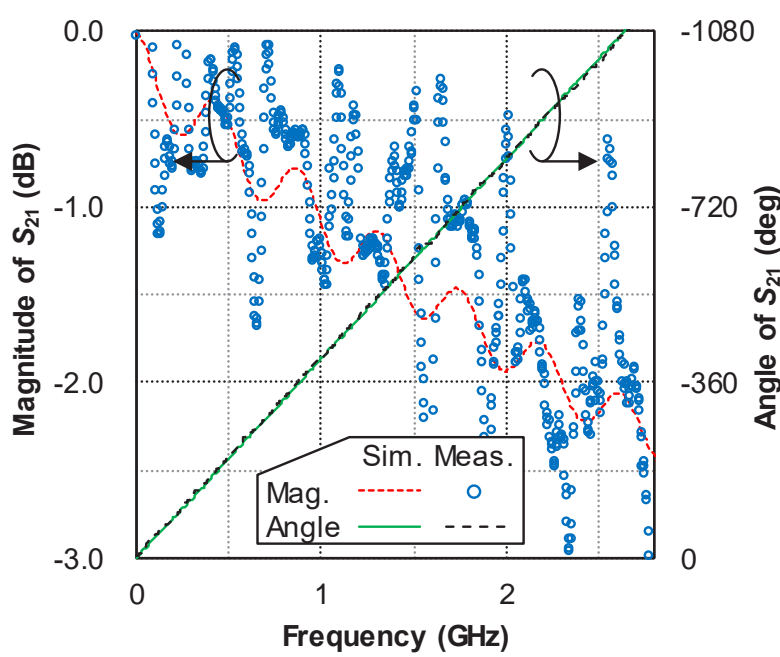

(a) 1.6-mm-wide transmission line

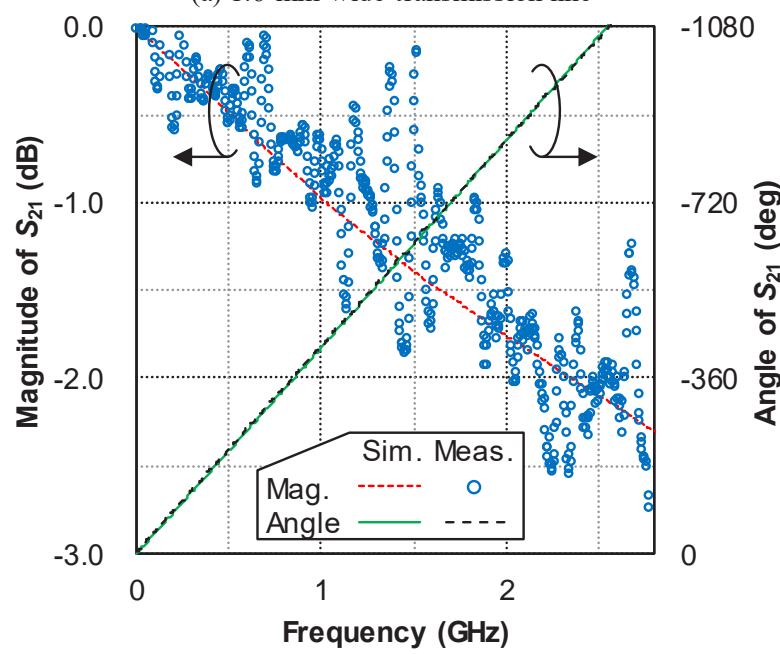

(b) 3.0-mm-wide transmission line

Fig. 5 Measured and simulated results of $S_{21}$ : $\varepsilon_{\text {q }}$ and $\tan \delta$ set to 0.43 and 0.023 , respectively.
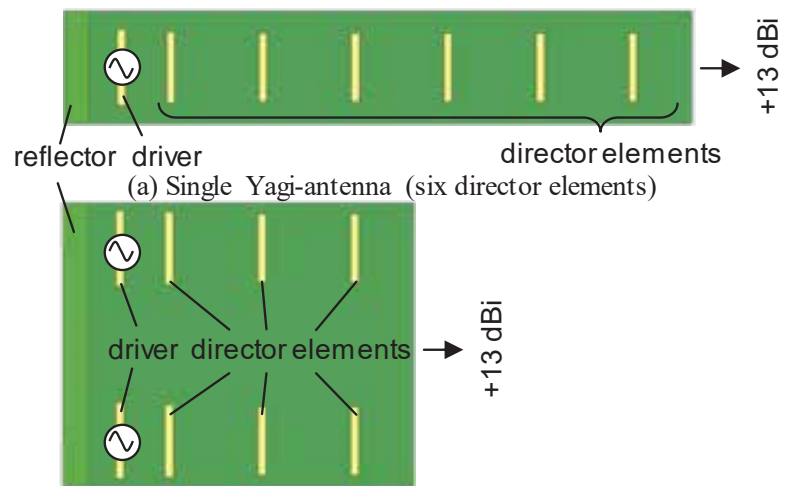

(b) Array Yagi-antenna (three director elements)

Fig. 6 Geometries of single and array Yagi-antennas on PCBs: The Yagi-antenna is composed of a reflector, a driver, and several director elements.

\section{Double Rat-Race Balun}

In an actual circuit, a power divider is necessary to feed the signal power to the array. A balun is also necessary to excite the two arms of the driver element. The balun is normally placed right after the power divider. Although the path from an RF port to antenna terminals requires (I) low insertion loss, (II) wide band, (III) fair balance, and (IV) isolation properties, the combination of the power divider and the balun deteriorates such requirements. Therefore, we proposed the use of the double rat-race balun not only as a power divider but also as a balun. Figure 8(a) shows the double rat-race balun for the array Yagi-antenna [12, 13]. The RF port (Port 1) signal is divided into the antenna ports (Ports 2 and 3 ) via microstrip lines. Ports 2 and 3 are differential ports because succeeding coplanar striplines prevent common signals. (I) Since the RF port is only connected to antenna ports with low-loss microstrip lines, the low insertion loss is maintained. In contrast, the combination of a balun and a power divider exhibits a sum of their losses in $\mathrm{dB}$. (II) The wideband property is inherited from the rat-race balun. In contrast, the combination of the power divider and the balun limits the bandwidth, which is an intersection of those. (III) Positive (+) and negative $(-)$ node signals should be the same magnitude with a 180degrees otherwise boresight of a single Yagi-antenna driven by $(+/-)$ nodes misaligned to the beam formed by the array antenna, as reported in [6]. This fair balance property is also inherited from the rat-race balun. (IV) Since the clockwise and counterclockwise lengths from port $2(+/-)$ to port $3(+/-)$ are $\lambda_{\mathrm{TL}}$ and $3 \cdot \lambda_{\mathrm{TL}} / 2$, respectively, the difference is $\lambda_{\mathrm{TL}} / 2$ (=180 degrees) and ports 2 and 3 are isolated when $\lambda_{\mathrm{TL}}$ is the wavelength on the microstrip line. Figure 8(b) is a TEG of the double rat-race balun. Figure 9 shows the measured $S_{21}, S_{31}$, and $S_{32}$. The RF signal is divided equally to antenna ports $\left(S_{21}, S_{31}\right)$ at the target frequency of $2.5 \mathrm{GHz}$. The phase difference between $S_{21}$ and $S_{31}$ is about 360 degrees from 2.0 to $3.0 \mathrm{GHz}$, and it shows that this double rat-race works as power divider and balun in this frequency range. The combination of a power divider and balun may possibly generate the quasi-differential signals of port $2(+/-)$ and port $3(+/-)$ but only the double rat-race balun satisfies all criteria (I) (IV) with a sacrifice of the footprint.

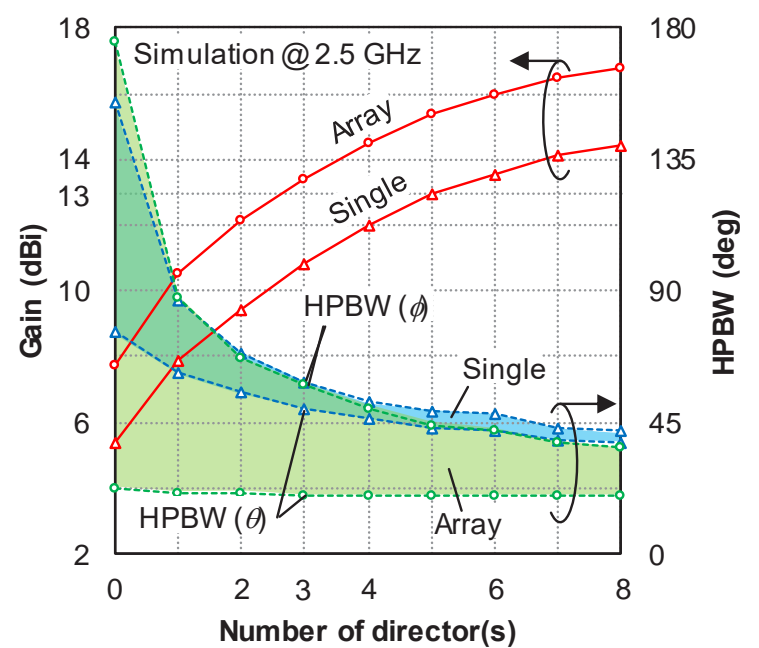

Fig. 7 Simulated gains of single and array Yagi-antennas with respect to the number of director elements: Azimuth $(\phi)$ and elevation $(\theta)$ angles of the half-power beamwidths (HPBWs) are also indicated. 


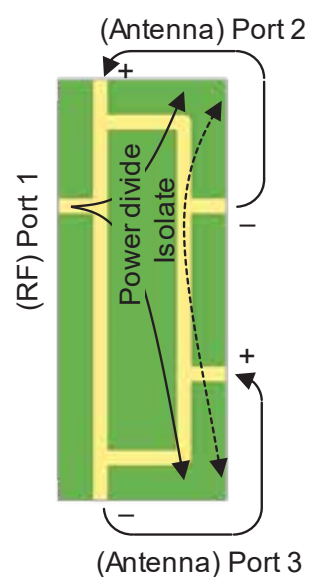

(a) Geometry

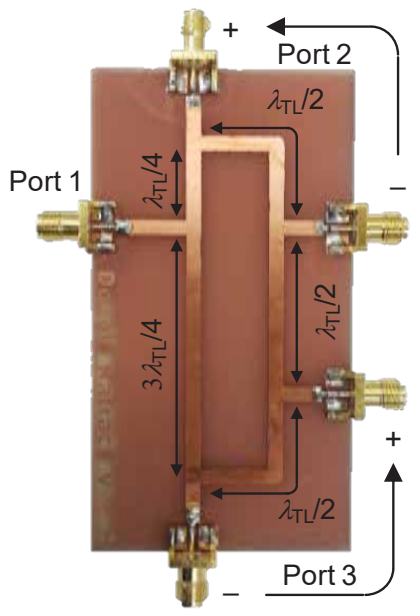

(b) TEG
Fig. 8 Geometry and TEG of the double rat-race balun: Positive $(+)$ and negative (-) nodes are assumed as differential ports because the nodes are combined with a coplanar stripline.
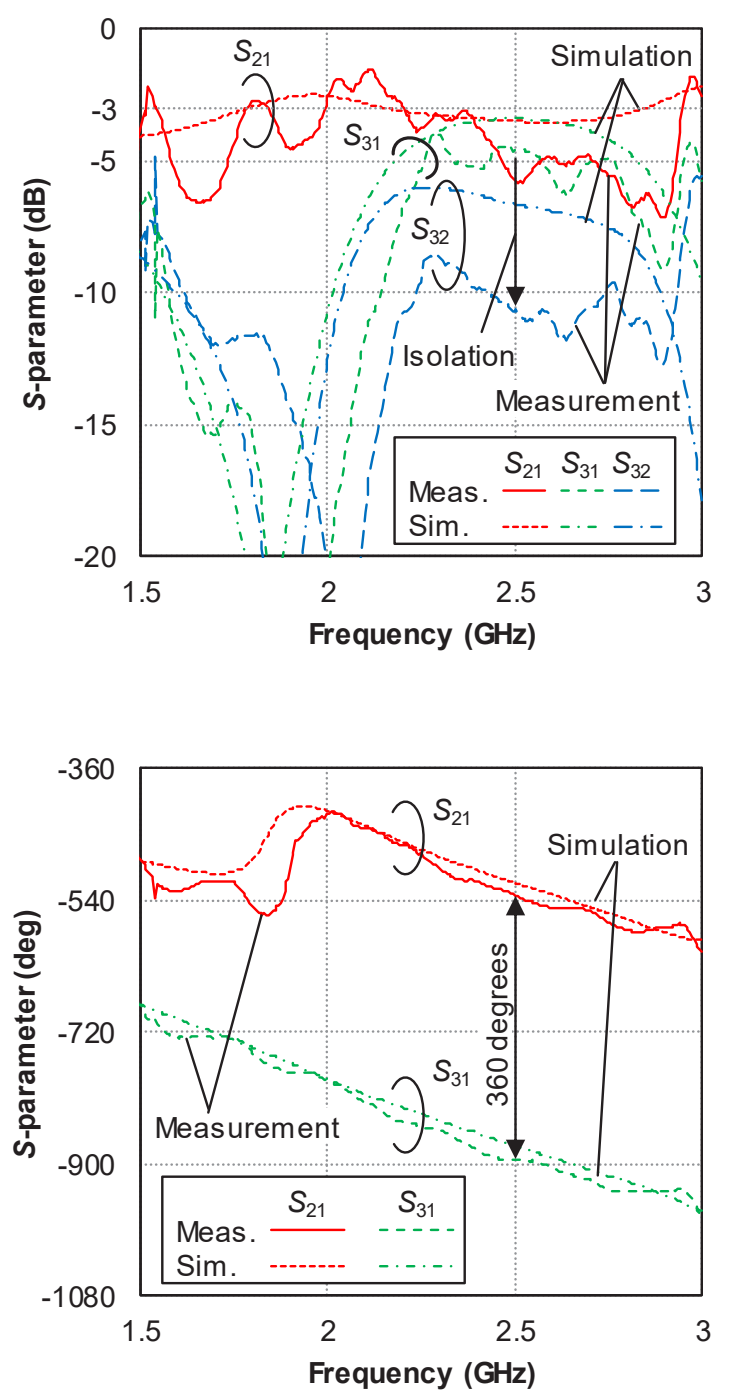

Fig. $9 S$-parameter of the double rat-race balun

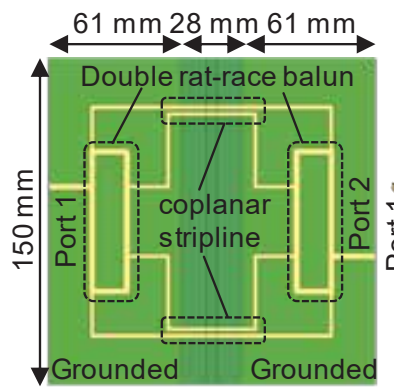

(a) Geometry for simulation

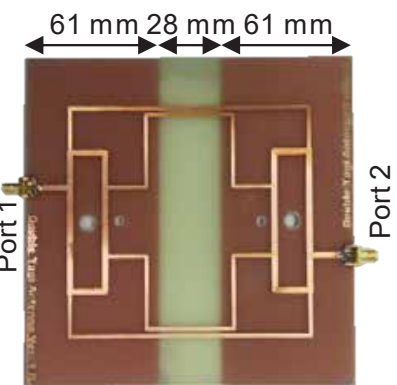

(b) TEG for measurement
Fig. 10 Geometry and TEG for the back-to-back simulation and measurement of the double rat-race balun: The double ratrace baluns are connected with coplanar striplines.

Figure 10(a) shows a back-to-back connection of the double rat-race balun to estimate the insertion loss. Two double rat-race baluns are connected via coplanar striplines. Figure 10(b) is a TEG for measurement. Figure 11 shows the measured back-to-back connection of the double rat-race balun. The insertion loss is half the $\left|S_{21}\right|$ of the TEG because $\left|S_{21}\right|$ contains two double rat-race baluns. The insertion loss is about $2 \mathrm{~dB}$ around the target frequency of $2.5 \mathrm{GHz}$. The reflection of $\left|S_{11}\right|$ is sufficiently low. Figure 12(a) shows the geometry of the coplanar stripline that bridges the left- and right-hand sides of the double rat-race baluns in Fig. 10. Figure 12(b) shows the simulated result of the coplanar stripline. The commonmode $S_{21}$ is greatly suppressed in comparison with that of the differential mode. Since the double rat-race balun is connected to the driver element of an antenna via this coplanar stripline, differential excitation is guaranteed. Although Takano et al. [12] used a differential amplifier, which has a nonreciprocal property, to suppress the common-mode signal, the reciprocal property $\left(S_{21}=S_{12}\right)$ of the coplanar stripline is required for communication (TRx) and functions under not only a transmitting antenna (Tx) but also a receiving one $(\mathrm{Rx})$.

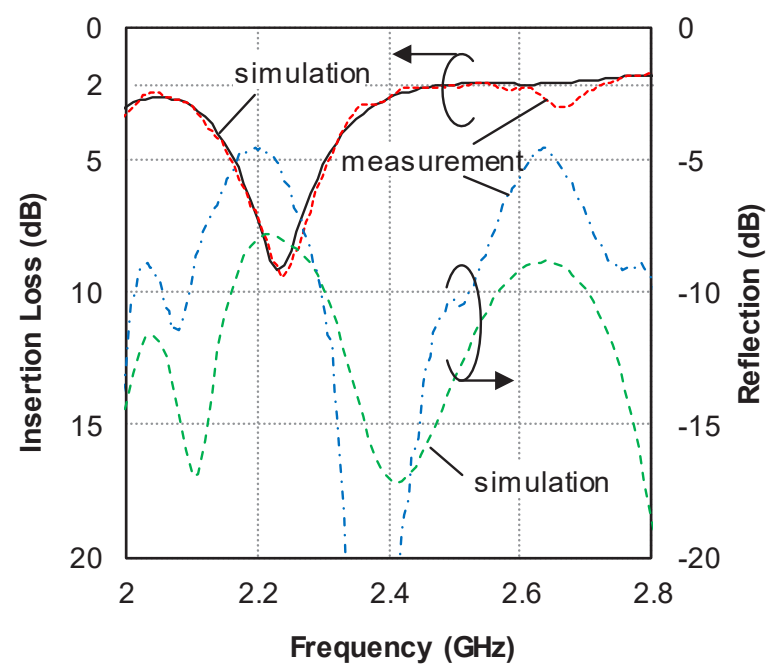

Fig. 11 Insertion loss and reflection coefficient of the double ratrace balun: The insertion loss is half the $\left|S_{21}\right|$ of the backto-back simulation/measurement in $\mathrm{dB}$. 


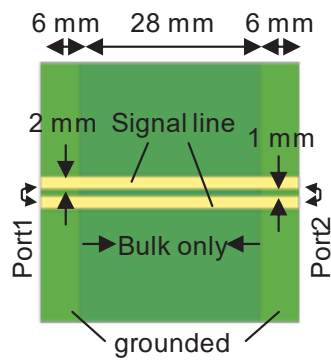

(a) Geometry

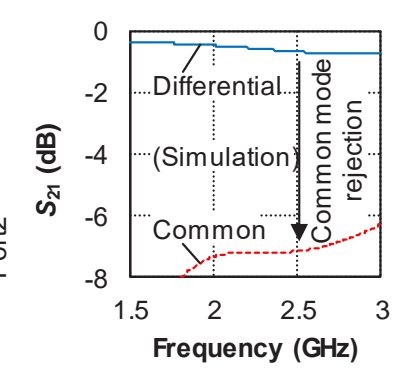

(b) Simulated result

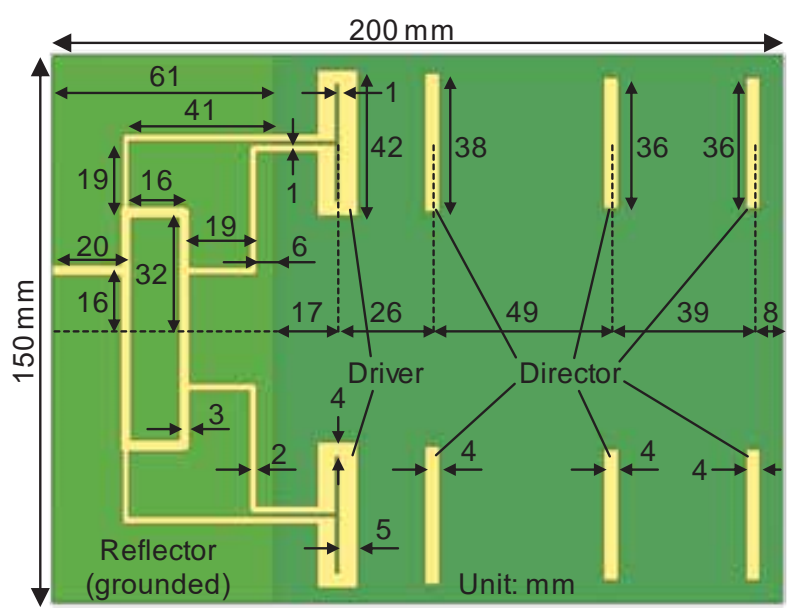

(a) Geometry

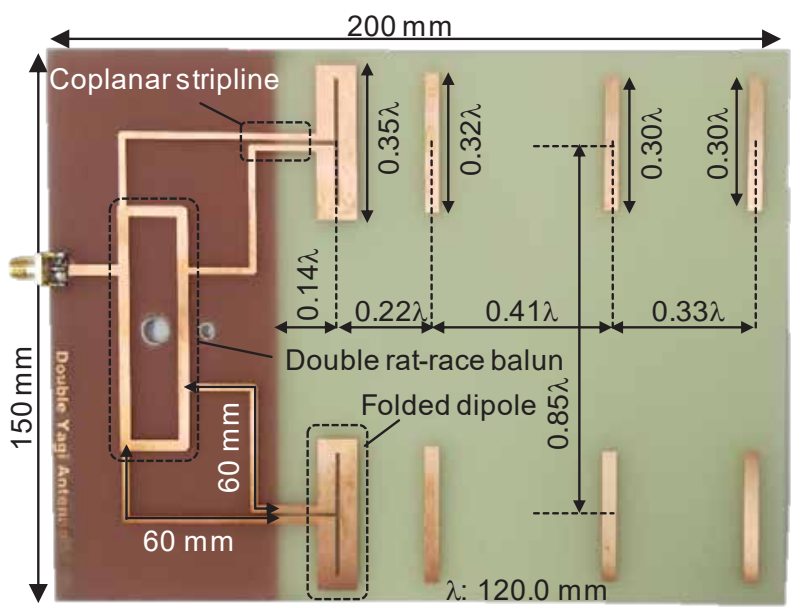

(b) Prototype

Fig. 13 The $1 \times 2$ array Yagi-antenna on a PCB

\section{Array Yagi-Antenna}

Figures 13(a) and (b) show the antenna geometry and its prototype. Since two single Yagi-antennas are placed in parallel, this antenna is a $1 \times 2$ array Yagi-antenna. The SMA (SubMiniature version A) connector is mounted on the left edge and it is an RF port of this antenna. The RF signal is divided into two differential signals with the double rat-race balun. The differential signals are connected to folded dipoles via equidistant microstrip lines and coplanar striplines. The folded dipole antennas are the driver elements of the Yagi-antenna. The ground plane of the microstrip lines also acts as the reflector element of the Yagi- antenna. Since the Yagi-antenna has three director elements, this Yagi-antenna has five elements in total, including the reflector and driver elements. Figure 14 shows the setup for the measurement of its propagation at $1 \mathrm{~m}$ distance. Figure 15 shows the measured result. The highest $S_{21}$ of $-18 \mathrm{~dB}$ is observed at around $2.5 \mathrm{GHz}$. The reflection coefficient is lower than $-10 \mathrm{~dB}$ from 2.1 to $2.5 \mathrm{GHz}$. The fractional bandwidth is about $16 \%$. At this band, group delay is flat.

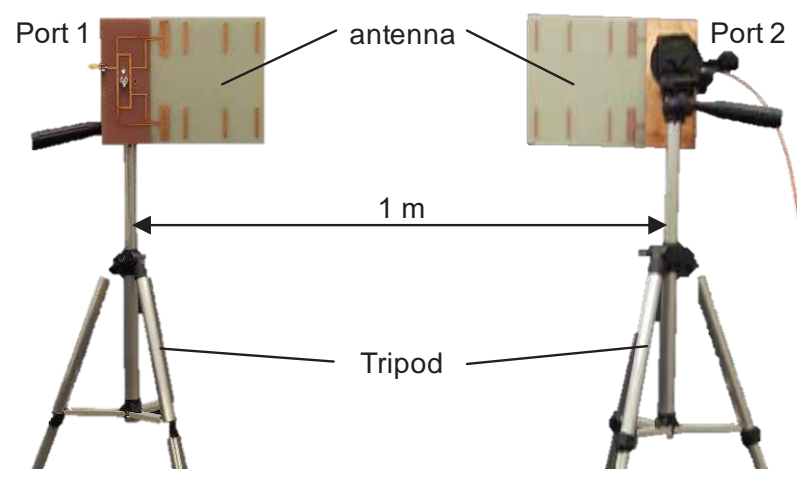

Fig. 14 Measurement setup of the $1 \times 2$ array Yagi-antenna

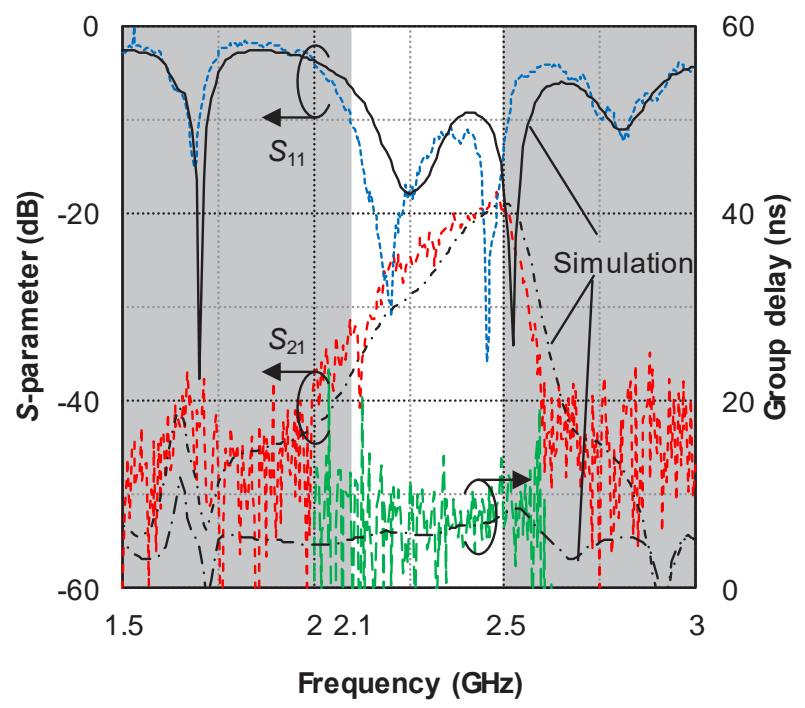

Fig. 15 Measured and simulated $S$-parameter and group delay of the $1 \times 2$ array Yagi-antennas $1 \mathrm{~m}$ away

Figure 16 shows the simulated radiation pattern of this $1 \times 2$ array Yagi-antenna at $2.5 \mathrm{GHz}$. The main lobe points along the $\mathrm{X}$-axis ( $\phi$ and $\theta$ are 0 and 90 degrees, respectively) and its simulated gain is $11.4 \mathrm{dBi}$. Figures $17(\mathrm{a})$, (b), and (c) show the normalized radiation patterns of electrical fields $|E|$ on $\mathrm{H}$ - and E-planes at 2.3, 2.4, and $2.5 \mathrm{GHz}$. The half-power beamwidths (HPBWs) are at the angles $\phi$ and $\theta$, where $|E|$ is greater than 0.707 . The HPBW at the azimuth angle $(\phi)$ is greater than that at the elevation angle $(\theta)$. Antenna directivity is estimated from these HPBWs, and the estimated values are plotted in Fig. 18 [15]. Since the antenna gain is a directivity degraded by insertion loss, the antenna gain is also plotted in Fig. 18 after subtracting the insertion loss of Fig. 11 from the directivity. As shown 
in this graph, the antenna gain is $13.0 \mathrm{dBi}$ at the target frequency of $2.5 \mathrm{GHz}$. The highest antenna gain is $14.1 \mathrm{dBi}$ at $2.53 \mathrm{GHz}$. Figure 19 shows the front-to-back ratio (F/B). $\mathrm{F} / \mathrm{B}$ achieves $20 \mathrm{~dB}$ at the target frequency of $2.5 \mathrm{GHz}$, which means the reflector element works effectively even though the reflector element is shared with the ground plane of the microstrip lines.

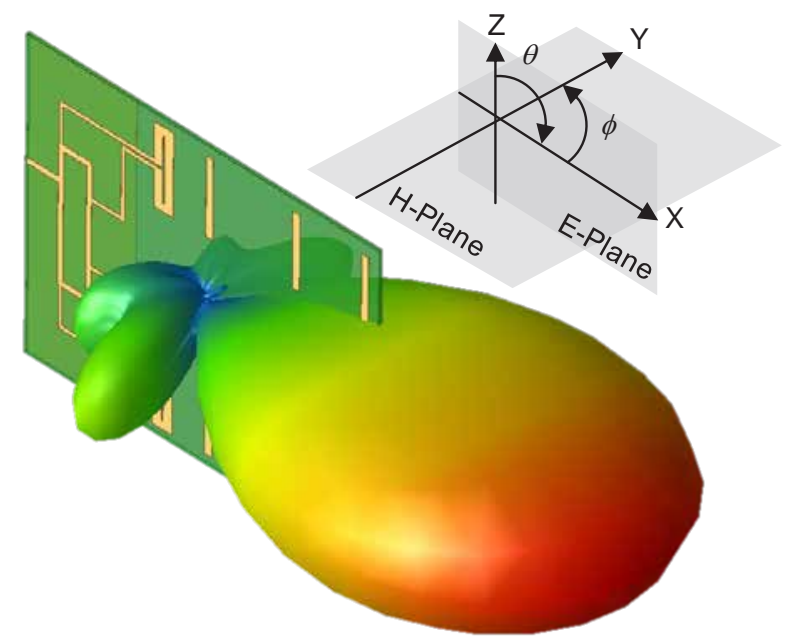

Fig. 16 Simulated radiation pattern of the $1 \times 2$ array Yagi-antenna at $2.5 \mathrm{GHz}$

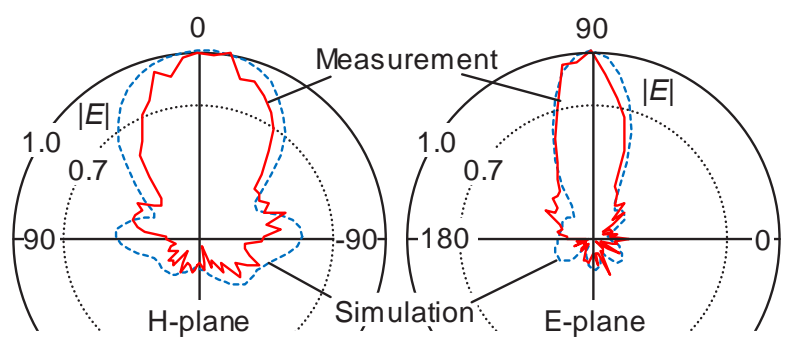

(a) $2.3 \mathrm{GHz}$

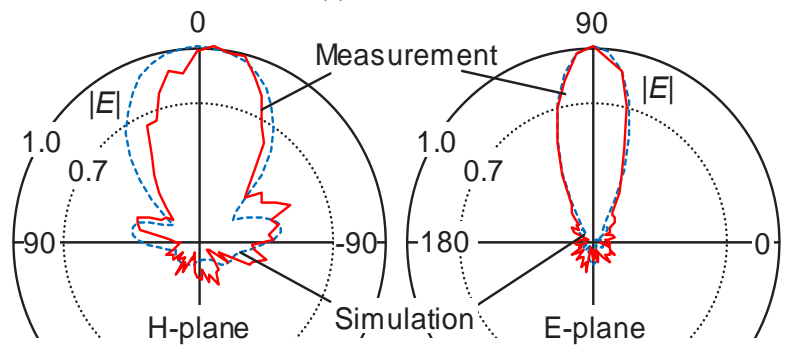

(b) $2.4 \mathrm{GHz}$

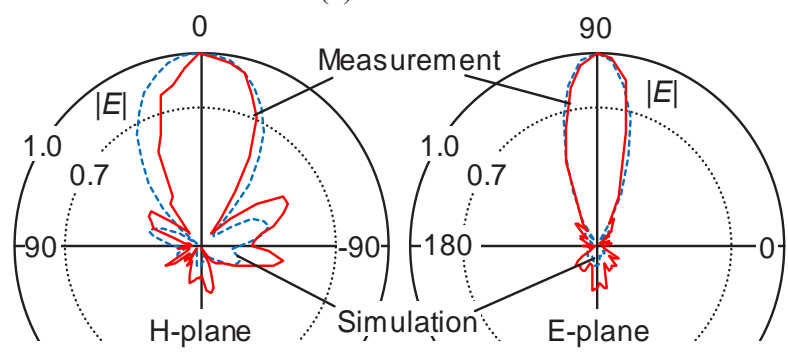

(c) $2.5 \mathrm{GHz}$

Fig. 17 Normalized radiation patterns of an electric field $|E|$ as functions of azimuth $\phi$ and elevation $\theta$ angles
Table 1 lists the features of reported $2.4 \mathrm{GHz}$ array Yagi-antennas. These antennas achieve high gains of +10 $\mathrm{dBi}$ using the array structure. The antenna gain in this work is comparable to those in other works even though its volume is the minimal $1.6 \times 200 \times 150 \mathrm{~mm}^{3}$ (48 cc). The band of this antenna covers from 2.1 to $2.5 \mathrm{GHz}$ because the double rat-race balun works as not only a power divider but also a balun. The reason why the double rat-race balun is adopted in the signal transformer to the antenna is mainly because of its wide bandwidth, which is essential for increasing the data rate in WDC. Although these desired antenna performances are based on the double ratrace balun properties of (I) low insertion loss, (II) wide band, (III) fair balance, and (IV) isolation, further investigation is necessary to clarify the contribution ratios. As a result, this antenna increases the number of computational nodes of WDC with a high-speed communication capability.

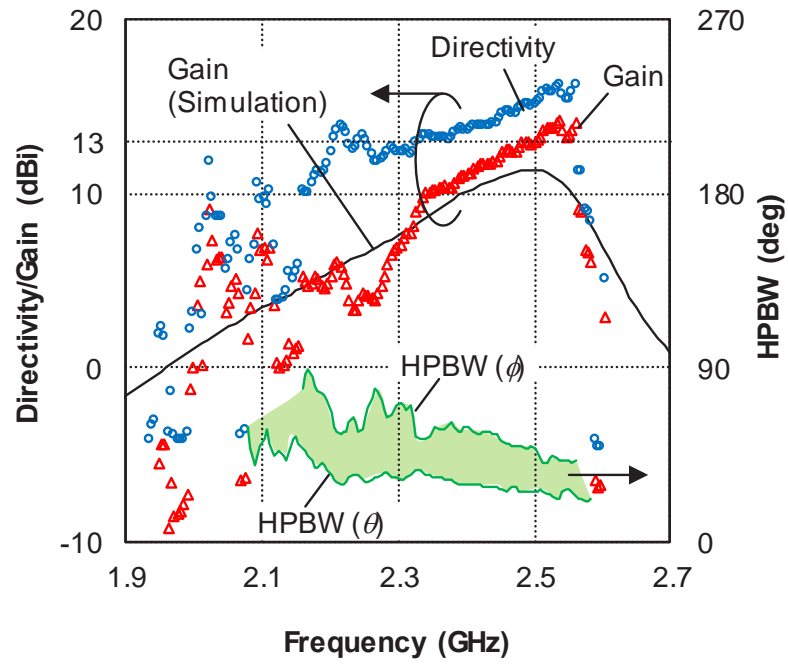

Fig. 18 Measured directivity, gain, and half-power beamwidth (HPBW) of the $1 \times 2$ array Yagi-antenna as a function of frequency

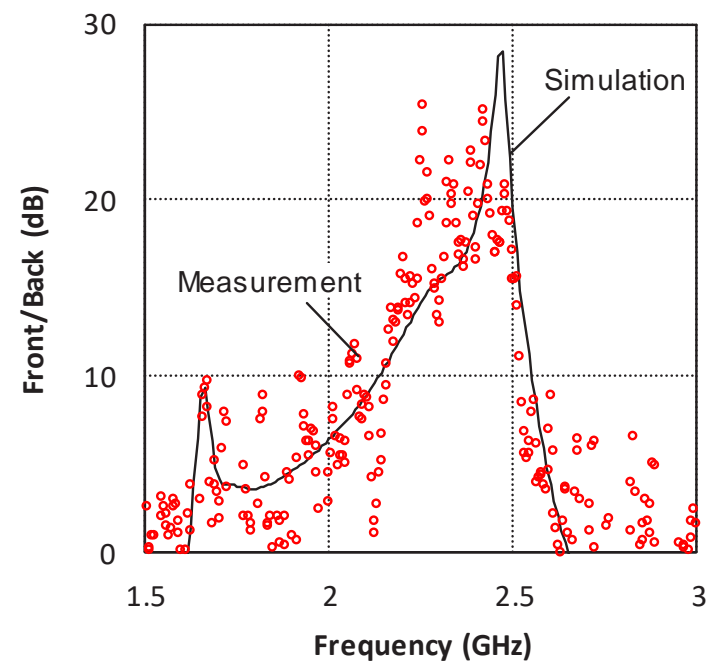

Fig. 19 Measured front-to-back ratio (F/B) of the $1 \times 2$ array Yagi-antenna as a function of frequency 
Table 1 Features of $2.4 \mathrm{GHz}$ array Yagi-antennas

*) Estimated from figures

\begin{tabular}{|c|c|c|c|c|c|}
\hline & [4] & [5] & \multicolumn{2}{|c|}{ [6] } & This work \\
\hline Elements & 4 & 4 & \multicolumn{2}{|l|}{8} & 5 \\
\hline Array & $1 \times 4$ & $1 \times 4$ & $1 \times 8$ & $4 \times 8$ & $1 \times 2$ \\
\hline Substrate & $1 \mathrm{~mm}-\mathrm{FR} 4$ & $1 \mathrm{~mm}-\mathrm{FR} 4$ & \multicolumn{2}{|c|}{$1.524 \mathrm{~mm}$ - Rogers 4350B } & $1.53 \mathrm{~mm}-\mathrm{FR} 4$ \\
\hline Balun & MS-DSPS & MS-DSPS & \multicolumn{2}{|c|}{ MS-CPS } & \\
\hline Power Divider & Wilkinson & Wilkinson & \multicolumn{2}{|c|}{ Microstrip } & Double rat-race \\
\hline Band $(\mathrm{GHz})$ & 2.45 & $2.37-2.54^{*}(6.9 \%)$ & $2.36-2.45^{*}(3.8 \%)$ & 2.4 & $2.1-2.5(16.0 \%)$ \\
\hline Gain (dBi) & 11.5 & 12 & 15.41 & 18 & 13.0 \\
\hline $\mathrm{F} / \mathrm{B}(\mathrm{dB})$ & $25^{*}$ & 15 & $20^{*}$ & $20^{*}$ & 20.0 \\
\hline HPBW $\left(^{\circ}\right)$ & $45^{*}$ & 80 & 47 & 25 & 48.2 \\
\hline Size $\left(\mathrm{mm}^{3}\right)$ & $\begin{array}{c}56 \times 150 \times 150^{*} \\
(1,260 \mathrm{cc}) \\
\end{array}$ & $\begin{array}{c}1 \times 160 \times 320^{*} \\
(51 \mathrm{cc})\end{array}$ & $\begin{array}{c}4.6 \times 243 \times 550 \\
(615 \mathrm{cc})\end{array}$ & $\begin{array}{c}190 \times 243 \times 550 \\
(25,394 \mathrm{cc})\end{array}$ & $\begin{array}{c}1.6 \times 200 \times 150 \\
(48 \mathrm{cc})\end{array}$ \\
\hline
\end{tabular}

MS-DSPS: microstrip-to-double-sided parallel stripline, MS-CPS: microistrip-to-coplanar stripline

However, the peak gain of $14.1 \mathrm{dBi}$ is exhibited at 2.53 $\mathrm{GHz}$, which is the outside of this antenna band. Therefore, the higher gain can be obtained by further tuning the antenna elements without changing the antenna structure. Although the insertion loss of $2 \mathrm{~dB}$ seems high compared with the enhancement due to the array configuration, the commercial single $2.4 \mathrm{GHz}$ Yagi-antenna on the FR-4 PCB has a comparable insertion loss of $1.44 \mathrm{~dB}$ at its center frequency [16]. We expect that the insertion loss of 2 $\mathrm{dB}$ and the footprint can be diminished by applying the spline-shaped wiring of the microstrip lines [10].

\section{Wireless Distributed Computing (WDC)}

WDC is a technology that enhances the computational speed by connecting computational nodes over a wireless network [1]. For instance, low-cost internet-of-things (IoT) devices collaborate to compute the complex image processing locally on the WDC and then upload the result to a cloud server. As a result, computation latency, power consumption, and communication data size are reduced. In this paper, we demonstrate WDC using this $1 \times 2$ array Yagi-antenna. Figure 20 shows the spectrum of the IEEE $802.11 \mathrm{~b}$ known as Wifi. Wifi has 13 channels. Their center frequencies are allocated from 2412 to $2472 \mathrm{MHz}$ in 5 $\mathrm{MHz}$ steps. Since the wideband property of this antenna covers these channels, the USB-Wifi device (Realtek RTX8811AU) is attached to this antenna. Note that the Wifi transceiver should be able to cover the total antenna band simultaneously to enable the use of the entire band. Since this Wifi channel occupies only $20 \mathrm{MHz}$, a 20-timeshigher data rate is possible when utilizing the $400 \mathrm{MHz}$ band $(2.1-2.5 \mathrm{GHz})$. A laptop PC (Panasonic CF-RZ4, Intel M-5Y70 processor) is connected to this USB-Wifi device via a USB cable. After installing a Microsoft Message Passing Interface (MS-MPI) and a $\pi$ estimation program to three PCs [17], they are connected to the antennas via USB-Wifi devices [13]. Three antennas are placed $1 \mathrm{~m}$ away and the $\pi$ estimation program is executed; then, its calculation time is observed. Since the custom-design antenna requires an outdoor license, the distance is limited to $1 \mathrm{~m}$ even though the system is designed to work over a long distance. This long-distance feature and HPBW denoted in Table 1 decide the coverage area of computational nodes outdoors. MS-MPI is a framework to combine computer resources, and the $\pi$ estimation program is a Monte Carlo simulator compiled with Microsoft Visual Studio. Figure 21 shows the duration time of the $\pi$ estimation program with respect to the number of threads. Since the M5 Y70 processor has 2-core 4-threads, the maximum number of threads is twelve with three PCs. The duration time decreases with the number of threads. A wired connection using 1-Gb Ethernet instead of the antenna is also examined, as shown in Fig. 21. Although the wireless connection has some overhead in comparison with the wired one, the acceleration factors are in good agreement between the two cases. The acceleration factor of twelve threads reaches $550 \%$ in comparison with that of one thread. This result shows that this antenna realizes WDC, which reduces computational latency.

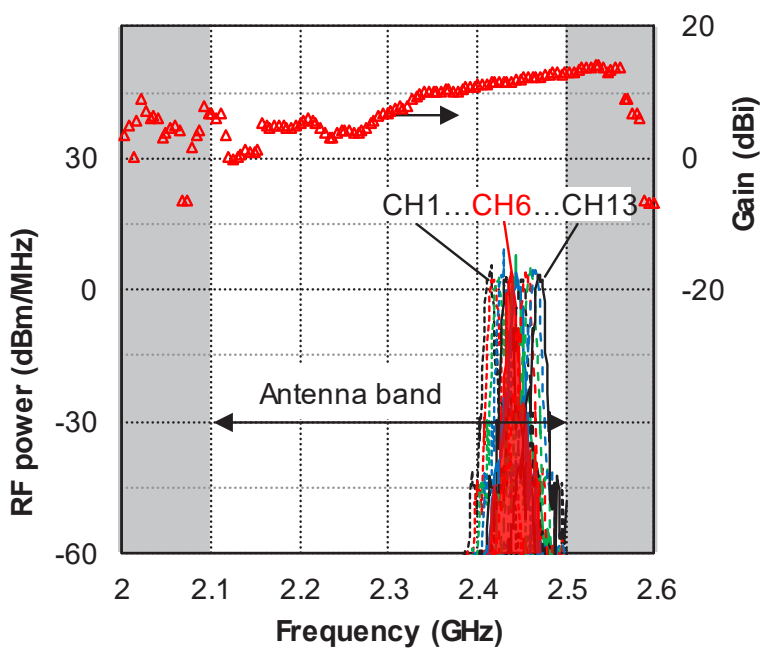

Fig. 20 Spectrum of the USB-Wifi (IEEE 802.11b) unit: This spectrum is measured in the peak-hold mode. 


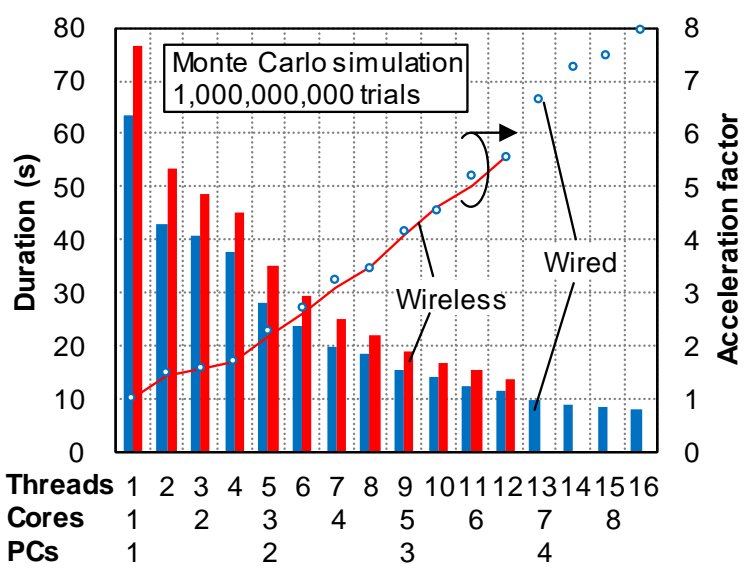

Fig. 21 Duration of 1,000,000,000-iteration Monte Carlo simulations for $\pi$ estimation over wired and wireless networks: The connections are $1 \mathrm{~Gb}$ Ethernet and IEEE 802.11b (CH6), respectively. Each PC (CF-RZ4) contains the Intel 5 Y70 processor having 2-core 4-threads.

\section{Conclusion}

We evaluated the performance of the wireless distributed computing $($ WDC) system with the $1 \times 2$ array Yagiantenna. We extracted the substrate parameters of relative permittivity and loss tangent to enable accurate electromagnetic simulations. We compared gains and radiation patterns of single and array Yagi-antennas. The radiation pattern of the array Yagi-antenna was suitable for WDC. Since the array Yagi-antenna required a power divider and balun, we introduced the double rat-race balun, which works not only as a power divider but also as a balun. We confirmed that the power distribution of the double ratrace balun is ideally performed with low insertion loss. We developed a prototype of the $1 \times 2$ array Yagi-antenna. Its bandwidth was broad in comparison with other array Yagiantennas that use the cascade connection of a power divider and balun. We measured a high gain of the antenna despite its compactness. We applied this antenna for WDC. As a result, the acceleration factor of twelve threads was $550 \%$ in comparison with that of one thread. Using this antenna, we hope to increase the number of computational nodes over a long distance in heterogeneous processors.

\section{References}

[1] D. Datla, X. Chen, T. Tsou, S. Raghunandan, S. M. S. Hasan, J. H. Reed, C. B. Dietrich, T. Bose, B. Fette and J.-H. Kim: Wireless distributed computing: A survey of research challenges, IEEE Commun. Mag., Vol. 50, No. 1, pp. 144-152, 2012.

[2] H. Yagi: Beam transmission of ultra-short waves, Proc. IRE, Vol. 16, No. 6, pp. 715-740, 1928.

[3] R.-N. Cai, M. C.-Yang, S. Lin, X.-Q. Zhang, X.-Y. Zhang and X.F. Liu: Design and analysis of printed Yagi-Uda antenna and twoelement array for WLAN applications, Int. J. Antennas Propag., Vol. 2012, pp. 1-8, 2012.

[4] S. C. Lin, C. W. Hsieh, C. H. Chang and T. L. Jong: Design and implementation of planar rectenna for ISM-band application, 45th European Microwave Conf., pp. 999-1002, 2015.

[5] Y. Song, J. Wang and X. Luo: Design of a high gain quasi-Yagi antenna and array for rectenna, Proc. IEEE Int. Symp. on Antennas and Propagation, pp. 1089-1090, 2017.

[6] C. Lee, H. Park, Y. Lim and S. Kahng: A stacked array antenna able to make a very large gain and tilt the end-fire beam at the ISMband, Int. J. of RF and Microwave Computer-Aided Engineering, pp. 1-7, 2019

[7] C. Lee, C. Lin, C. Tsai and C. Wang: Broadband printed-circuit Yagi array, 5th Int. Conf. on Wireless Communications, Networking and Mobile Computing, pp. 1-4, 2009.

[8] F. C. Costa, G. Fontgalland, A. G. D Assunção, T. P. Vuong and L. M. Mendonca: A new quasi-Yagi bowtie type integrated antenna, Int. Telecommunications Symp., pp. 468-471, 2006.

[9] M. Farran, S. Boscolo, A. Locatelli, A.-D. Capobianco, M. Midrio, V. Ferrari and D. Modotto: Compact quasi-Yagi antenna with folded dipole fed by tapered integrated balun, Electron. Lett., Vol. 52, No. 10, pp. 789-790, 2016.

[10] F. Weinmann: Planar 9-element quasi-Yagi antenna array for Xband application, The European Conf. on Wireless Technology, pp. 539-542, 2005.

[11] R. A. Alhalabi and G. M. Rebeiz: Differentially-fed millimeterwave Yagi-Uda antennas with folded dipole feed, IEEE Trans. on Antennas and Propagation, Vol. 58, No. 3, pp. 966-969, 2010.

[12] K. Takano, S. Amakawa, K. Katayama, S. Hara, R. Dong, A. Kasamatsu, I. Hosako, K. Mizuno, K. Takahashi, T. Yoshida and M. Fujishima: A $105 \mathrm{~Gb} / \mathrm{s} 300 \mathrm{GHz}$ CMOS transmitter, IEEE Int. Solid-State Circuits Conf., pp. 308-309, 2017.

[13] K. Katayama, T. Baba and T. Ohsawa: 13.6 dBi stacked-Yagi antenna on a PCB for edge computing, RISP Int. Workshop on Nonlinear Circuits, Communications and Signal Processing, pp. 363-366, 2020.

[14] D. C. Laney: Modulation, Coding and RF Components for UltraWideband Impulse Radio, Ph.D. thesis, University of California, 2003.

[15] J. Kraus and R. Marhefka: Antennas, McGraw-Hill Education, 1950.

[16] R. Wallace and S. Dunbar: $2.4 \mathrm{GHz}$ YAGI PCB Antenna, Texas Instrument Application Note DN034, 2010.

[17] P. Pacheco: Parallel Programming with MPI, Morgan Kaufmann, 1996.

\section{Acknowledgments}

This work is supported by the Japan Society for the Promotion of Science (JSPS) through a Grant-in-Aid for Scientific Research (No. 18K04155). This work is also supported by the VLSI Design and Education Center (VDEC), The University of Tokyo, in collaboration with Agilent Technologies. 


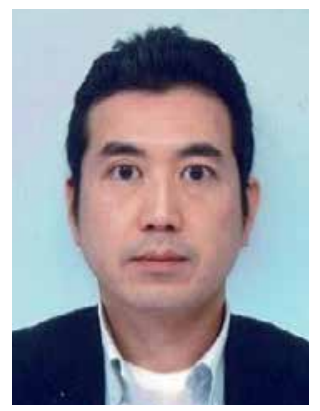

Kosuke Katayama received his B.E. and M.E. degrees in electronics engineering from Hosei University in 1997 and 1999, respectively. He received his $\mathrm{Ph} . \mathrm{D}$. degree in materials engineering from Hiroshima University in 2003. After working at an RF simulator company, he joined Hiroshima University as a researcher in 2010. He has been an Assistant Professor of Waseda University since 2017. He is a member of RISP, JSAP, IEICE and IEEE.

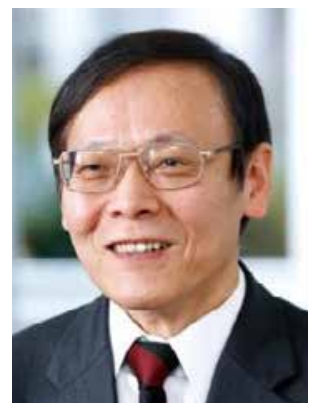

Takaaki Baba received his M.S. degree and Dr. of Engineering degree from Nagoya University in 1973 and 1979, respectively. He joined Matsushita Electric Industrial Co., Ltd., in 1973. Between 1983 and 2002, he worked at Matsushita Electric Co. in the U.S. where he was involved in several strategic projects, such as system LSI and ASIC applications, wireless communication systems and electronic devices. He was a Research Fellow at UC Berkeley between 1980 and 1982 and at Stanford University between 2002 and 2003. From 2003 to March -2019, he was a Professor in the field of integrated systems at the Graduate School of Information, Production and Systems of Waseda University. Now he is a Professor Emeritus at Waseda University. He is a member of IEEE and served as an Executive Committee member of IEEE-ISSCC from 1995 to 2003 . He is a member of RISP and IEICE. He also serves as an Associate Editor of JSP (Journal of Signal Processing).

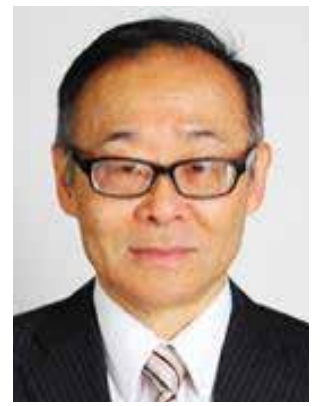

Takashi Ohsawa rceived his B.S. and M.S. degrees in physics from Waseda University, Tokyo, Japan, in 1977 and 1979, respectively, and Ph.D. degree in electronic engineering from the University of Tsukuba, Tsukuba, Japan, in 2009. During 1982-2010, he worked on research and development of semiconductor memories at the Semiconductor Device Engineering Laboratory, Toshiba Corporation, Kawasaki and Yokohama, Japan. From 2010 to 2014, he was a Professor at the Center for Spintronics Integrated Systems, Tohoku University, Sendai, Japan. Since 2017, he has been a Professor with the Graduate School of Information, Production and Systems, Waseda University, Kitakyushu, Japan.

(Received May 10, 2020; revised September 1, 2020) 